\title{
OPERATOR PARAMETERIZATIONS OF FRAME GENERATORS AND GENERALIZED DUAL PAIR OF FRAME GENERATORS OF UNITARY SYSTEMS
}

\author{
XUNXIANG GUO
}

Abstract. In this paper, we first give a condition such that a countable unitary system has complete wandering vectors, which plays the key role to operator parameterize frame generators for unitary systems in the literatures. Then we continue to study more general operator parameterizations and their applications. Based on the invertibility of some parameterizing operators, we introduce the concept of generalized dual pair of frame generators for a unitary system, which is a natural generalization of dual pair of frame generators. Then we characterize generalized dual pair of frame generators and some interesting properties and constructions of generalized dual frame generator pairs are also studied.

Mathematics subject classification (2010): 42C15, 46B15.

Keywords and phrases: Unitary system, operator parameterization, frame generator, generalized dual pair of frame generators.

\section{REFERENCES}

[1] C. K. ChuI, An Introduction to Wavelets, Acad. Press, New York, 1992.

[2] I. DAubechies, Ten Lectures on Wavelets, CBMS 61, SIAM, 1992.

[3] X. DAI, D. LARSON, Wandering vectors for unitary systems and orthogonal wavelets, Mem. Amer. Math. Soc. 134 (640) (1998).

[4] R. J. Duffin, A. C. Shaffer, A class of nonharmonic Fourier Series, Trans. Amer. Math. Soc., 72 (1952), 341-366.

[5] J. P. GABARDO, D. HAN, Frame representations for group-like unitary operator systems, J. Operator Theory, 49 (2003), 1-22.

[6] X. GuO, Wandering r-tuples for unitary systems, J. Math. Anal. Appl. 374 (2011) 722-728.

[7] X. Guo, Multi-frame vectors for unitary systems, Indian J. Pure Appl. Math., 43 (4), 2012, 391-409.

[8] D. Han AND D. LaRson, Bases, frames and group representations, Memoirs of Amer. Math. Soc., Vol. 147, No. 697, Sep. 2000.

[9] D. HAN, Wandering vectors for irrational rotation unitary systems, Trans. Amer. Math. Soc. 350 (1998), 309-320.

[10] D. HAN, D. LARSON, Wandering vector multipliers for unitary groups, Trans. Amer. Math. Soc. 353 (2001), 3347-3370.

[11] E. Hernandez And G. Weiss, A First Course on Wavelets, CRC press, Boca Raton, FL, 1996.

[12] S. MALLAT, Multiresolution approximations and wavelet orthonormal basis of L2 (R), Trans. Amer. Math. Soc. 315 (1989), 69-87.

[13] B. Sz-NAGY, Expansion Theorems of Paley-Wiener type, Duke Math. J., 14 (1947), 975-978. 